\title{
Evaluation of the Antioxidant Activity of Crude Whole Plant Methanolic Extract of Oxalis stricta Linn
}

\author{
Raphael Dzinyela ${ }^{1,}$, , Abdul-Nasir Abdul-Baasit ${ }^{2}$, Abdul Razak Alhassan ${ }^{1}$ \\ ${ }^{1}$ College of Biology and Environment, Nanjing Forestry University, Nanjing, China \\ ${ }^{2}$ Department of Molecular Biology and Genetics, Mugla Sttk1 Kocman University, Mugla, Turkey
}

\section{Email address:}

dzinyelar@gmail.com (R. Dzinyela), abdulbaasit17@yahoo.com (Abdul-Nasir Abdul-Baasit),

nanakwame3356@gmail.com (A. R. Alhassan)

${ }^{*}$ Corresponding author

\section{To cite this article:}

Raphael Dzinyela, Abdul-Nasir Abdul-Baasit, Abdul Razak Alhassan. Evaluation of the Antioxidant Activity of Crude Whole Plant Methanolic Extract of Oxalis stricta Linn. American Journal of Applied Chemistry. Vol. 9, No. 2, 2021, pp. 43-48.

doi: 10.11648/j.ajac.20210902.11

Received: May 9, 2021; Accepted: May 20, 2021; Published: May 27, 2021

\begin{abstract}
Globally, it is well-known for some time now that the oxidation in vegetables and organisms is significant; it is responsible for cells' existence. The side effects are free radicals, reactive oxygen species (ROS), and reactive nitrogen species. Every living organism has an antioxidant defense and maintenance system that enables it to handle the ROS produced. In a biological system or an organism, an imbalance of ROS and antioxidant capacity leads to a phenomenon called oxidative stress. Naturally, antioxidant defense and maintenance systems of most biological systems or organisms are unable to cater to the entire oxidative injury. Oxidative stress is known to result in chronic diseases, including autoimmune diseases, cancer, etc. Oxalis stricta Linn. is a valuable medicinal plant native to Florida and uses many diseases. This study was intended to assess antioxidant activity, evaluate the phenol contents, and screen for phytonutrients present in $O$. stricta Linn. The antioxidant activity was determined by 2, 2-Diphenyl-1-picrylhydranyl-hydrate (DPPH) assay and phosphomolybdate assay with ascorbic acid as reference antioxidant, while the phenol content was also determined using Folin-Ciocalteu assay. Phytochemical screening revealed that Oxalic acid, flavonoids, tannins, phenols, and glycosides were present. The total phenol content present in the crude methanolic extract was $25.26 \pm 1.02 \mathrm{mg}$ gallic acid equivalent/g of dried extract. Antioxidant activity of extract by DPPH assay was expressed as $\mathrm{IC}_{50}$ values $(\mu \mathrm{g} / \mathrm{ml})$; the $\mathrm{IC}_{50}$ of the extract was $205.10 \mu \mathrm{g} / \mathrm{ml}$ and $85.04 \mu \mathrm{g} / \mathrm{ml}$ for ascorbic acid. The total antioxidant activity of the extract using phosphomolybdate assay was $24.73 \pm 1.14 \mathrm{mg}$ ascorbic acid equivalent/g of dried extract. Therefore, the results suggest that the phenolic content of $O$. stricta Linn. provides substantial antioxidant activity. The authors recommend further investigations for the isolation and then the characterization of specific active components present in $O$. stricta Linn.
\end{abstract}

Keywords: Oxalis stricta Linn, Antioxidant Activity, Phytochemical Analysis, Phenol Content

\section{Introduction}

Globally, it is well-known for some time now that the oxidation in vegetables and organisms is significant; it is responsible for cells' existence. The side effects are the production of free radicals, reactive oxygen species (ROS), and reactive nitrogen species; these include hydroxyl radicals, singlet oxygen, hydrogen peroxide, and superoxide radicals. Although energy production, phagocytosis, stimulation of cell growth, intercellular signaling, and the formulation of biological compounds are the most beneficial roles played by the ROS. Every living organism has an antioxidant defense and maintenance system that enables it to handle the ROS produced. These antioxidant defense and maintenance systems can be exogenous or endogenous based on origin. In a biological system or an organism, an imbalance of ROS and antioxidant capacity leads to a phenomenon called oxidative stress. Naturally, antioxidant defense and maintenance systems of most biological systems or organisms are unable to cater to the entire oxidative injury. Therefore, oxidative stress results in chronic diseases such as autoimmune diseases, cancer, 
neurodegenerative diseases, aging, atherosclerosis, diabetes mellitus, arthritis, and inflammations [1-3].

Antioxidants serve as free radical scavengers, metal chelators, reducing agents, singlet molecules quenchers, and antioxidant enzyme activators which reduce the injury caused by the free radicals in the biological systems. Not long ago, synthetic antioxidant supplements, which include butylated hydroxyl toluene (BHT), butylated hydroxylanisole (BHA), tertiary butyl hydroquinone (TBHQ), and propylgallate (PG) were recognized to suppress oxidative injury. However, based on their carcinogenic and liver toxicity effects, they are no more in use. Nowadays, much interest has been given to the study of the antioxidant activity of plants. Lately, it is established that plant products like polyphenolic compounds (e.g., tannins and flavonoids) and various extracts of plants possess antioxidant potential. Therefore, dietary antioxidant supplement is the potential booster for these defense and maintenance systems [4-6].

Oxalis stricta Linn. (yellow wood sorrel) is native to North America, British Columbia, and Florida. It spreads steadily and is now available across the globe. It is mainly identified by its narrow, yellow-green rim of slender, white fur. Slender stalks and their rounded leaves support their flowers and fruits. It is also recognized to have some medicinal properties like febrifuge, stomachic, antiemetic, and astringent. Whole plant infusion is for fever, indigestion, inflammation externally, loss of appetite, mouth irritations, nausea, and stomach cramps; leaf poultice is for external bleeding treatment; root decoction is also for blood toxicity treatment $[7,8]$. Therefore, this study seeks to evaluate the antioxidant activity of crude whole plant methanolic extract of $O$. stricta Linn. It uses different methods, namely, the 2, 2-Diphenyl-1picrylhydrazyl (DPPH) radical scavenging method, phosphomolybdate assay for total antioxidant activity, and Folin-Ciocalteu assay for total phenol contents.

\section{Materials and Methods}

\subsection{Chemicals and Reagents}

All reagents and solvents from different suppliers were of the highest purity needed for each application. They include the 1, 1-Diphenyl-2-picrylhydrazl (DPPH), sodium phosphate, ammonium molybdate, sulphuric acid, FolinCiocalteu reagents, ethanol, Ascorbic acid, gallic acid, and sodium carbonate.

\subsection{Plant Material}

The whole plant of Oxalis stricta Linn. was obtained from Kwame Nkrumah University of Science and Technology, Ghana (KNUST). Mr. Raphael Ackah in the Department of Pharmacognosy, KNUST identified and authenticated it.

\subsection{Preparation of Plant Extract}

For extraction (methanol), $25 \mathrm{~g}$ of the whole plant $O$. stricta Linn. dried samples were ground into a fine powder in a mill and were mixed with $100 \mathrm{~mL}$ of $70 \%(\mathrm{v} / \mathrm{v})$ methanol.
The macerated mixture was permitted to stand for 72 hours at room temperature $\left(20^{\circ} \mathrm{C}\right)$. The extraction solvent became colored (brown). The obtained extract was filtered over Whitman No. 1 paper, and the filtrate was collected, then the solvent was removed by a rotary evaporator at $50^{\circ} \mathrm{C}[1]$.

\subsection{Qualitative Phytochemical Screening}

The qualitative phytochemical tests of crude whole plant methanolic extract of $O$. stricta Linn. was performed based on the following standard methods $[9,10]$.

\subsubsection{Tests for Carbohydrates}

Iodine test: To the test solution, $2 \mathrm{ml}$ of iodine solution was added. Dark blue or purple colors indicated that carbohydrates were present.

\subsubsection{Test for Tannins and Phenols}

Ferric Chloride Test: To the test solution, $2 \mathrm{ml}$ of $2 \%$ solution of $\mathrm{FeCl}_{3}$ was added. Blue-green or black color proved that phenols and tannins were present.

\subsubsection{Tests for Flavonoids}

Alkaline reagent test: To the test solution, $2 \mathrm{ml}$ of $2 \%$ $\mathrm{NaOH}$ solution was added; intensive yellow color formed, which then turned colorless upon addition two drops of diluted acid; this result proved that flavonoids were present.

\subsubsection{Test for Saponins}

Froth Test: To the test solution, $5 \mathrm{ml}$ of distilled water was added to a test tube and shook vigorously. The foam formation proved that saponins were present.

\subsubsection{Test for Proteins}

Biuret Test: To the test solution, $4 \% \mathrm{NaOH}$ solution was added, followed by drops of $1 \% \mathrm{CuSO}_{4}$ solution; violet color appearance indicated that proteins were present.

\subsubsection{Test for Organic Acids}

Oxalic Acid: To the test solution, few drops of $\mathrm{KMnO}_{4}$ and dilute $\mathrm{H}_{2} \mathrm{SO}_{4}$ were added. Color disappearance proved that Oxalic acid was present.

Malic Acid: To the test solution, few drops of $40 \%$ of $\mathrm{FeCl}_{3}$ solution were added; the appearance of yellowish color indicated that Malic acid was present.

\subsubsection{Test for Glycosides}

Salkowski's test: To the test solution, about $2 \mathrm{ml}$ of $\mathrm{H}_{2} \mathrm{SO}_{4}$ concentrated was added. A reddish-brown color formed showed the entity of steroidal aglycone part of the glycoside.

Keller Killiani Test (Cardiac glycosides): $0.4 \mathrm{ml}$ of glacial acetic acid with a trace amount of ferric chloride was added to $0.5 \mathrm{~g}$ of the crude extract. Transferred into a small test tube; gently added a conc-sulphuric acid sideways of the test tube. The appearance of blue color in the acetic layer proved that cardiac glycosides were present.

\subsection{DPPH Radical Scavenging Capacity}

The antioxidant activity was measured based on radical scavenging of hydrogen donating ability using the stable 
radical DPPH. The test was performed based on a standard procedure with a slight modification [11]. The radical reduction was followed by a decline in the absorbance at 517 $\mathrm{nm}$. A volume of $2 \mathrm{~mL}$ of $1 \mathrm{mM}$ DPPH solution was added to $2 \mathrm{~mL}$ of methanolic solution of the extracts in test tubes. The tubes were capped with parafilm and then kept for one hour in the dark. Absorbance at $517 \mathrm{~nm}$ was measured with a spectrophotometer UV-Win and compared to an ascorbic acid calibration curve. Each assay was performed in triplicate. The DPPH radical percentage inhibition was determined using the formula:

$$
\text { DPPH inhibition }(\%)=\frac{A 0-A}{A 0} \times 100
$$

Where $\mathrm{A} 0=$ absorbance of control sample $(\mathrm{t}=0 \mathrm{~h})$ and $\mathrm{A}$ $=$ absorbance of a tested sample at the end of the reaction $(\mathrm{t}=$ $1 \mathrm{~h})$. The amount of sample necessary to decrease the absorbance of DPPH by $50 \% \quad\left(\mathrm{IC}_{50}\right)$ was calculated graphically for the methanolic solution of the O. stricta Linn. In five different concentrations.

\subsection{Total Phenolic Content}

The number of total phenols was evaluated by using the Folin-Ciocalteu method [12]. The test mixture was formed from $5.0 \mathrm{~mL}$ of distilled water, $0.5 \mathrm{~mL}$ of extract, and $0.5 \mathrm{~mL}$ of the Folin-Ciocalteu reagent in this procedure. After 3 minutes, $1.0 \mathrm{~mL}$ of a saturated sodium carbonate solution was added. This mixture was shaken and permitted to stand for $1 \mathrm{~h}$. The absorbance was measured at $725 \mathrm{~nm}$ with a spectrophotometer UV-Win. A gallic acid calibration curve was prepared. Total phenolic content was expressed as $\mu \mathrm{g}$ of gallic acid equivalents (GAE) per $\mathrm{mL}$; it was determined using the formula, $y=0.8725 x-1.2239$, where $x$ represents the amount of gallic acid equivalent $(\mu \mathrm{g} / \mathrm{mL})$, and $y$ represents the absorbance at $725 \mathrm{~nm}$.

\subsection{Total Antioxidant Activity}

The antioxidant activity of methanolic extract of $O$. stricta Linn. was determined by phosphomolybdate assay using ascorbic acid as a standard with a slight modification [13]. A volume of $1 \mathrm{ml}$ of reagent solution $(4 \mathrm{mM}$ ammonium molybdate, $0.6 \mathrm{M}$ sulphuric acid, and $28 \mathrm{mM}$ sodium phosphate) was mixed with $0.1 \mathrm{ml}$ of sample solution. The tubes were covered and incubated for 90 minutes at $95^{\circ} \mathrm{C}$ in a water bath. A typical blank with an appropriate volume of the reagent solution and the solvent was also incubated under similar conditions. After the samples had cooled to room temperature and using ascorbic acid as standard, the absorbance of the mixture was measured with a spectrophotometer UV-Win at $765 \mathrm{~nm}$ against a blank. An ascorbic acid calibration curve was prepared. Total antioxidant capacity was stated as $\mu \mathrm{g}$ of ascorbic acid equivalents (AAE) per $\mathrm{mL}$; it was determined using the formula, $y=0.9533 x-1.0139 x$ represents the amount of ascorbic acid equivalent $(\mu \mathrm{g} / \mathrm{mL})$, and $y$ represents the absorbance at $765 \mathrm{~nm}$.

\subsection{Statistical Analysis}

The results are stated as the mean of five measurements \pm standard deviation. Descriptive statistical analysis was performed using Microsoft Excel and/or Graph Pad Prism version 5.01 (Graph Pad Software Inc., La Jolla, CA, USA).

\section{Results}

\subsection{Phytochemical Screening}

Table 1. Results of Phytochemical screening tests.

\begin{tabular}{lll}
\hline S. No. & Phytonutrients & O. stricta Linn \\
\hline 1 & Carbohydrates & + \\
2 & Tannins and Phenols & + \\
3 & Flavonoids & + \\
4 & Saponins & - \\
5 & Proteins & - \\
6 & Organic Acids & + \\
7 & Glycosides & + \\
\hline
\end{tabular}

Legend: $(+)=$ Present; $(-)=$ Absent.

\subsection{DPPH Radical Scavenging Capacity}

DPPH was used to assess the ability of crude extract of $O$. stricta Linn. to serve as a free radical scavenger by evaluating the $\mathrm{EC}_{50}$ (lower value shows higher radical scavenging power). As shown in Table 2, the $\mathrm{IC}_{50}$ values for O. stricta Linn. extract and ascorbic acid are $205.10 \mu \mathrm{g} / \mathrm{ml}$ and $85.04 \mu \mathrm{g} / \mathrm{ml}$, respectively. In addition, it was observed that ascorbic acid demonstrated a higher antioxidant activity than that of the crude extract. Graphically, the relationship between the extract and ascorbic acid in DPPH scavenging capacity is also presented in Figure 1.

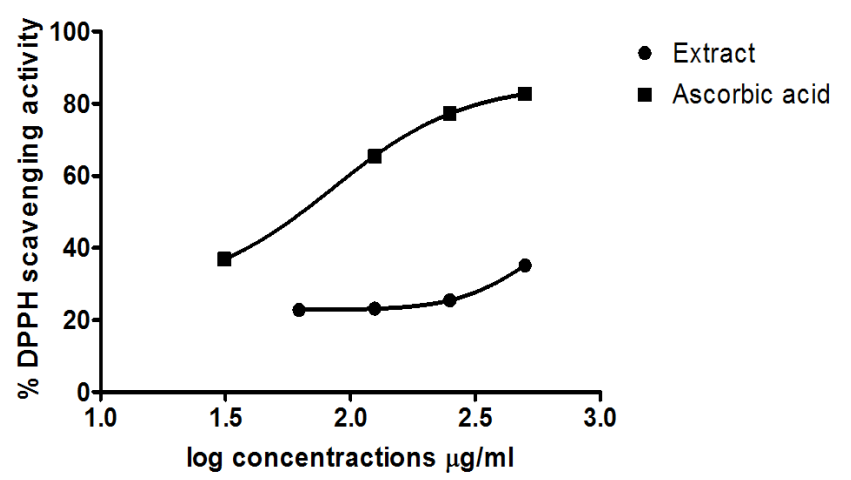

Figure 1. Free radical scavenging activities of total crude extract of $O$. stricta Linn. measured using the DPPH assay. Ascorbic acid was used as a reference antioxidant in this experiment.

\subsection{Total Phenolic Content}

Phenolic compounds are a large and diverse class of secondary metabolites of plants, and their significant sources in the human diet are various beverages, fruits, and vegetables. Same bioactive polyphenols, for example, proanthocyanidins, derivatives of hydroxycinnamic acids and flavonoids, are also present in forest trees. The flavonoids are best studied and the largest group among polyphenols [14, 
15]. To determine the potential antioxidant capacity of the crude extract of $O$. stricta Linn, it was necessary to evaluate the content of polyphenols in crude methanolic extract. The total phenols content expressed as Gallic acid equivalents has been determined for the extract spectrophotometrically, and the results are presented in Table 2 . The results also prepared a gallic acid calibration curve with $\mathrm{r}^{2}=0.6666$ (Figure 2). The data shows that natural antioxidant phenolic compounds are present in crude methanolic extract.

Table 2. Phenolic content, total antioxidant capacity, and DPPH scavenging activity of methanolic extract of O. stricta Linn.

\begin{tabular}{llll}
\hline & \multicolumn{2}{l}{ Antioxidant Capacity (Ascorbic Acid as standard) } & Total Phenolic Content \\
\cline { 2 - 4 } & DPPH Assay EC $\mathbf{5 0}_{\mathbf{5 0}}$ value $(\boldsymbol{\mu g} / \mathbf{m l})$ & Phosphomolybdate Assay AAE $(\mathbf{m g} / \mathbf{g} \pm$ SD) & GAE (mg/g士 SD) \\
\hline Extract & 205.10 & $2473 \pm 114.10$ & $2526 \pm 10.45$ \\
Ascorbic acid & 85.04 & - & - \\
\hline
\end{tabular}

Data are presented as mean $\pm \mathrm{SD}$.

*AAE=Ascorbic Acid Equivalent

$* \mathrm{GAE}=$ Gallic Acid Equivalent.

\subsection{Total Antioxidant Activity}

Total antioxidant capacity (TAC) of methanolic extract of $O$. stricta Linn. was determined by phosphomolybdate assay. The assay is based on the fact that the molybdenum (VI) is reduced to molybdenum $(\mathrm{V})$ in the presence of an antioxidant (reducing agent), leading to the formation of a green phosphomolybdate (V) complex that can be determined spectrophotometrically at $765 \mathrm{~nm}$. The assay thermally involves the generation of auto-oxidation at a higher temperature during a prolonged period of incubation. The assay deals with a mechanism of electron transfer (ET), and this reduction may be caused by some natural products, including phenols and flavonoids [16, 17]. The results, expressed as ascorbic acid equivalent (AAE), indicated a significant antioxidant activity of methanolic extract (Table 2.), which may be attributed to phenolic compounds present in the extract [13]. An ascorbic acid calibration curve with $r^{2}=0.5748$ was also prepared from the results (Figure 3 ).

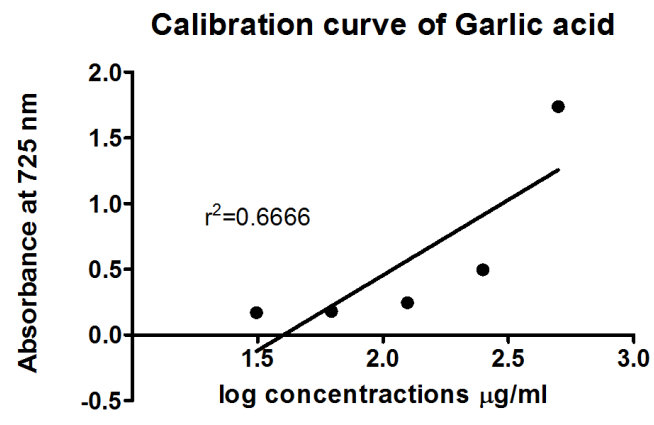

Figure 2. A gallic acid calibration curve.

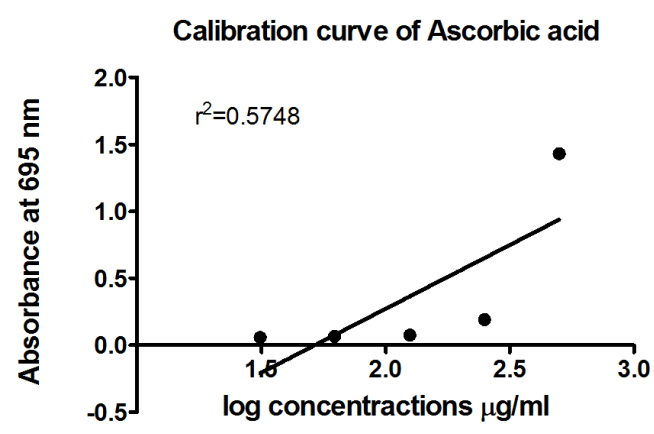

Figure 3. An ascorbic acid calibration curve.

\section{Discussion}

\subsection{Phytochemical Screening}

Phytochemical analysis of crude whole plant methanolic extract of $O$. stricta Linn. indicated the active phytochemical constituents such as Oxalic acid, glycosides, phenols, and flavonoids, as shown in Table 1.

\subsection{DPPH Radical Scavenging Capacity}

Almost 50 years ago, Marsden Blois recognizably introduced the DPPH method used widely to test compounds' ability to serve as hydrogen donors or free radical scavengers and determine antioxidant capacity [11]. DPPH is a stable free radical which accepts hydrogen radical or an electron to become a stable diamagnetic compound $[18,19]$. The results obtained from the DPPH method are usually interpreted using the parameter $\mathrm{IC}_{50}$ ("efficient concentration" value) and may be described as the substrate concentration that causes loss of $50 \%$ of the DPPH activity (color). A lower $\mathrm{IC}_{50}$ value signifies higher radical scavenging capacity [20]. The DPPH method was used to evaluate the antioxidant activity of $O$. stricta Linn. in comparison with a known natural antioxidant, ascorbic acid. Ascorbic acid showed a higher radical scavenging capacity, while crude extract of $O$. stricta Linn. was much lower. The methanolic extract of $O$. stricta Linn. and ascorbic acid were evaluated concerning their $\mathrm{IC}_{50}$ values (Table 2). The crude extract $\mathrm{IC}_{50}$ value $(205.10 \mu \mathrm{g} / \mathrm{ml})$ is about three times higher than ascorbic acid $(85.04 \mu \mathrm{g} / \mathrm{ml})$. Other plant studies have shown similar results [21, 22]. The extract of $O$. stricta Linn. demonstrated a significant free radical scavenging activity; this may result from the synergistic antioxidant activity of several phytonutrients present in the crude methanolic extract, which include phenols, flavonoids, and others [4, 23].

\subsection{Total Phenolic Content}

Phenolic compounds are a large and diverse group of secondary metabolites of plants, and their significant sources in the human diet are various beverages, fruit, and vegetables. The flavonoids are best studied and the largest group among polyphenols $[14,15]$. Phenolic compounds may 
be either simple, single aromatic-ringed, low molecularweight compounds, complex tannins, or derived polyphenols. There is a significant relationship between phenolic content and total antioxidant activity of selected grain products, fruits, and vegetables. The phenolic compounds possess antioxidant capacity mainly by their redox properties; this enables them to function as singlet oxygen quenchers, reducing agents, metal chelators, or hydrogen donors. Phenols have also shown multiple physiological and medicinal functions in both plants and humans. Phenolic compounds also undertake a prominent preventive role in heart diseases, aging-related diseases, and cancer development $[4,24,25]$. The results of phenols content of methanolic extract of $O$. stricta Linn. was stated as gallic acid equivalents using the calibration curve equation: $y=$ $0.8725 \mathrm{x}-1.2239, \mathrm{r}^{2}=0.6666$, where $\mathrm{x}$ represents total phenolic content in the crude extract of $O$. stricta Linn. expressed in $\mathrm{mg} / \mathrm{g}$, and y represents absorbance at $725 \mathrm{~nm}$ (Table 2). It indicated that natural antioxidant phenolic compounds are present in the methanolic extract. Similar results have also been reported in plant studies [20]. The results showed that phenolic compounds contributed significantly to the potential antioxidant activity of the $O$. stricta Linn. [26].

\subsection{Total Antioxidant Capacity}

Total antioxidant capacity (TAC) of methanolic extract of O. stricta Linn. was determined by phosphomolybdate assay. The assay is established on the detail that the molybdenum (VI) is reduced to molybdenum (V) in the presence of an antioxidant (reducing agent), leading to the formation of a green phosphomolybdate (V) complex that can be determined spectrophotometrically at $765 \mathrm{~nm}$. The assay deals with a mechanism of electron transfer (ET), and this reduction may be caused by some natural products, including phenols and flavonoids [16, 17]. Therefore, a higher absorbance value of crude extract shows more vital antioxidant capacity. Furthermore, the results showed that the antioxidant activity of crude extract of $O$. stricta Linn. was concentration-dependent and expressed as ascorbic acid equivalent (AAE) (Table 2). Therefore, the potent antioxidant activity of crude extract of $O$. stricta Linn. may be due to the presence of its polyphenolic compounds [13].

\section{Conclusion}

The study proved that $O$. stricta Linn. possesses considerable antioxidant capacity. There was also a significant correlation between antioxidant activity and phenolics, substantiating the recognized free radical scavenging capacity of $O$. stricta Linn. The prominent scavenging potential of $O$. stricta Linn. may be attributable to hydroxyl groups present in the phenolic and its derivative compounds. These findings validate the potentials of $O$. stricta Linn. as a vital source of naturally occurring antioxidants and support the use of whole plant infusion for fever, indigestion, inflammation externally, loss of appetite, mouth irritations, nausea, and stomach cramps; leaf poultice is for external bleeding treatment; root decoction is also for blood toxicity treatment.

\section{Recommendation}

Having established the antioxidant capacity of whole plant extract of O. stricta Linn, this study recommends that there be a further investigation for the isolation and then the characterization of specific active components present in Oxalis stricta Linn.

\section{Conflict of Interest Statement}

The authors declare that they have no competing interests.

\section{Acknowledgements}

The authors are grateful to University for Development Studies, Tamale, for implementing this study. In addition, Mr. Raphael Ackah and Mr. Edward Amankwah were acknowledged for their support in the sampling of plants.

\section{References}

[1] Keser, S., et al., Hydrogen peroxide radical scavenging and total antioxidant activity of hawthorn. Chem J, 2012.2 (1): p. 9-12.

[2] Chang, S.-T., et al., Antioxidant activity of extracts from Acacia confusa bark and heartwood. Journal of Agricultural and Food Chemistry, 2001. 49 (7): p. 3420-3424.

[3] Antolovich, M., et al., Methods for testing antioxidant activity. Analyst, 2002. 127 (1): p. 183-198.

[4] Victor, B. K. and A. S. Hamed, WMA, (2014) Preliminary Phytochemical Screening and Evaluation of in vitro antioxidant activity of Iraqi species of Hypericum perforatum arieal part. International research journal of pharmacy. 5 (5): p. 369-373.

[5] Rahman, M., et al., Antioxidant activity of Centella asiatica (Linn.) Urban: Impact of extraction solvent polarity. Journal of Pharmacognosy and Phytochemistry, 2013. 1 (6): p. 27-32.

[6] Kulisic, T., et al., Use of different methods for testing antioxidative activity of oregano essential oil. Food chemistry, 2004. 85 (4): p. 633-640.

[7] Shah, S., T. Dhanani, and S. Kumar, Comparative evaluation of antioxidant potential of extracts of Vitex negundo, Vitex trifolia, Terminalia bellerica, Terminalia chebula, Embelica officinalis and Asparagus racemosus. Innov. Pharm. Pharmacother, 2013. 1: p. 44-53.

[8] Patel, S. A., S. Rajendra, and S. R. Setty, Hepatoprotective activity of Oxalis stricta Linn. on paracetamol-induced hepatotoxicity in albino rats. Algerian J Nat Prod, 2016. 4: p. 233-40.

[9] Mamta, S. and S. Jyoti, Phytochemical screening of Acorus calamus and Lantana camara. International Research Journal of Pharmacy, 2012. 3 (5): p. 324-326. 
[10] Gul, R., et al., Preliminary Phytochemical Screening, Quantitative Analysis of Alkaloids, and Antioxidant Activity of Crude Plant Extracts from $<i>$ Ephedra intermedia $</ i>$ Indigenous to Balochistan. The Scientific World Journal, 2017. 2017: p. 5873648.

[11] Molyneux, P., The use of the stable free radical diphenylpicrylhydrazyl (DPPH) for estimating antioxidant activity. Songklanakarin J. sci. technol, 2004. 26 (2): p. 211219.

[12] Brighente, I., et al., Antioxidant activity and total phenolic content of some Brazilian species. Pharmaceutical Biology, 2007. 45 (2): p. 156-161.

[13] Saeed, N., M. R. Khan, and M. Shabbir, Antioxidant activity, total phenolic and total flavonoid contents of whole plant extracts Torilis leptophylla L. BMC Complementary and alternative medicine, 2012. 12 (1): p. 1-12.

[14] Nishaa, S., et al., Antioxidant activity of ethanolic extract of Maranta arundinacea L. tuberous rhizomes. Asian Journal of Pharmaceutical and Clinical Research, 2012. 5 (4): p. 85-88.

[15] Kasangana, P. B., P. S. Haddad, and T. Stevanovic, Study of polyphenol content and antioxidant capacity of Myrianthus arboreus (Cecropiaceae) root bark extracts. Antioxidants, 2015. 4 (2): p. 410-426.

[16] Ahmed, D., M. M. Khan, and R. Saeed, Comparative analysis of phenolics, flavonoids, and antioxidant and antibacterial potential of methanolic, hexanic and aqueous extracts from Adiantum caudatum leaves. Antioxidants, 2015.4 (2): p. 394-409.

[17] Phatak, R. S. and A. S. Hendre, Total antioxidant capacity (TAC) of fresh leaves of Kalanchoe pinnata. Journal of Pharmacognosy and Phytochemistry, 2014. 2 (5): p. 32-35.
[18] Babu, D., et al., Antioxidant and free radical scavenging activity of triphala determined by using different in vitro models. Journal of Medicinal Plants Research, 2013. 7 (39): p. 2898-2905.

[19] Kasabe, P. J., et al., Nutritional, elemental analysis and antioxidant activity of garden cress (Lepidium sativum L.) seeds. Int. J. Pharm. Pharm. Sci, 2012. 4 (3): p. 392-395.

[20] Anthony, K. P. and M. A. Saleh, Free radical scavenging and antioxidant activities of silymarin components. Antioxidants, 2013. 2 (4): p. 398-407.

[21] Singh, S. K. and V. Prakash, Screening of antioxidant activity and phytochemicals strength of some herbal plants. International Journal of Pharmacy and Pharmaceutical Sciences, 2013.5 (3): p. 296-300.

[22] Shyur, L.-F., et al., Antioxidant properties of extracts from medicinal plants popularly used in Taiwan. Int J Appl Sci Eng, 2005. 3 (3): p. 195-202.

[23] Gardeli, C., et al., Essential oil composition of Pistacia lentiscus L. and Myrtus communis L.: Evaluation of antioxidant capacity of methanolic extracts. Food chemistry, 2008. 107 (3): p. 1120-1130.

[24] Soengas, P., et al., Functional plant science and biotechnology. Global science books, 2011. 2: p. 43-55.

[25] Omoregie, E. and A. Osagie, Antioxidant properties of methanolic extracts of some Nigerian plants on nutritionallystressed rats. Nigerian Journal of Basic and Applied Sciences, 2012. 20 (1): p. 7-20.

[26] Proestos, C., et al., Antioxidant capacity of selected plant extracts and their essential oils. Antioxidants, 2013. 2 (1): p. $11-22$. 